\title{
The regular and Mach reflections of shock waves from explosions in real air
}

\author{
F. Zhou, Z. Xu \& Y. G. Yu \\ Beijing Canbao Architecture Design Institute, Beijing, China
}

\begin{abstract}
Systematic research was carried out for shock wave reflection problems from an explosion in real air. When shock waves from an explosion on the ground meet with the mountain terrain of positive slope, a single reflection appears. When an aerial explosion above-ground is presented, on basis of the interaction of the incident shock wave with the ground, continuous reflection is produced. If the explosion occurs in a tunnel, due to restriction of the narrow tunnel space, a complicated interaction of shock waves with the walls and reflected shock waves is produced. A calculation of regular and Mach reflection of shock waves from an explosion is conducted with real air state equations. In a regular reflection region, we adopt the variable $\gamma$ regular reflection theory and the reflectiontermination condition is controlled by the sonic ray angle. In a Mach reflection region, we adopt the Russian scientist Korotkov method to establish the linear analysis solution for a small slope angle and give the start condition for Mach reflection and the limit control condition of Mach reflection for different explosions. Our study leads to a curve diagram showing the relation of ground shock waves' reflection overpressure and dynamic pressure versus incident overpressure and incident angle.

Keywords: regular reflection of shock wave, Mach reflection of shock wave, explosion, real air state equations, interaction of shock wave with the walls.
\end{abstract}

\section{Introduction}

We define a strong airblast as an airblast whose overpressure is greater than or equal to $4 \mathrm{MPa}$. When the overpressure of an airblast is greater than $4 \mathrm{MPa}$, or when the air temperature is higher than 2000 Kelvin, nitrogen and oxygen 
molecules in the air will be dissociated. When the temperature goes higher than $8000 \mathrm{~K}$, air atoms will be ionized.

Therefore, the state equations of ideal gases are applicable only to lowstrength airblasts, while strong airblasts require real-air state equations. The maximum reflection coefficient is calculated to be 8 with the ideal-gas model; however, the overpressure coefficient of reflected airblast wave could be greater than 10 in the real air.

This paper presents a theoretical calculation method of the strong airblast reflection coefficient with a variable ratio of specific heat (see Table 1).

Table 1: $\quad$ Specific heat ratio of incident wave and reflection wave.

\begin{tabular}{|c|c|c|c|}
\hline$\Delta \mathrm{P}_{\mathrm{f}}(\mathrm{MPa})$ & $\gamma_{\mathrm{f}}$ & $\Delta \mathrm{P}_{\mathrm{r}}(\mathrm{MPa})$ & $\gamma_{\mathrm{r}}$ \\
\hline 0.5 & 1.4 & 2.203 & 1.4 \\
\hline 1 & 1.4 & 5.397 & 1.382 \\
\hline 2 & 1.39 & 12.73 & 1.359 \\
\hline 5 & 1.346 & 38.74 & 1.312 \\
\hline 10 & 1.300 & 91.43 & 1.297 \\
\hline 20 & 1.26 & 208.6 & 1.264 \\
\hline
\end{tabular}

The data in this table are the results of strict calculation based on normal reflection of real-air shock wave overpressure impacting on the wall.

Let

$\Delta \mathrm{P}_{\mathrm{f}}$ - incident overpressure

$\Delta \mathrm{P}_{\mathrm{r}}$ - reflection overpressure

$\gamma_{\mathrm{f}}$ - specific heat ratio of incident wave

$\gamma_{\mathrm{r}}$ - specific heat ratio of reflection wave

The $\gamma_{\mathrm{f}}$ of incident pressure and the $\gamma_{\mathrm{r}}$ of reflected pressure are approximately equal.

Simulation experiments demonstrated the reliability and correctness of those calculation results.

\section{Calculation of strong airblast wave regular reflection}

\subsection{Normal reflection for explosions in air}

The overpressure of a normally reflected airblast can be calculated with the following formula: 


$$
\Delta P_{r}=2 \Delta P_{f}+\frac{\frac{\gamma+1}{\gamma-1} \Delta P_{f}^{2}}{\Delta P_{f}+\frac{2 \gamma}{\gamma-1} P_{0}}
$$

where $\gamma$ is the variable ratio of specific heat of the air, which is dependent on the incident air blast wave overpressure.

\subsection{The function of the ratio of specific heat depending on the real air overpressure}

Parameters at the air blast front satisfy the following shock wave conditions:

$$
\begin{gathered}
D=\frac{\rho_{f} u_{f}}{\rho_{f}-1} \\
p_{f}-1=u_{f} D \\
\varepsilon_{f}-\varepsilon_{0}=\frac{1}{2}\left(P_{f}+1\right)\left(1-\frac{1}{\rho_{f}}\right)
\end{gathered}
$$

And the real-air state equations are given by American scholar Brode:

$$
\begin{gathered}
\varepsilon=\frac{1}{2} z(\mu-1) \\
T=z \cdot v \\
\mu=\mu_{0}+\mu_{1} \ln \rho \\
\mu_{1}=\frac{2 z \cdot v^{\prime}}{v+z v^{\prime}} \\
\mu_{0}=\mu_{0}(Z) \\
v=v(Z)
\end{gathered}
$$

For obtaining the solution to above-mentioned equations and specific heat ratio, air density iteration technique is utilized frequently. So, the relation between the ratio of specific heat and the real-air pressure can be obtained.

\subsection{Regular reflection for bursts in air}

The overpressure of regularly reflected airblast waves can be calculated with the following formula.

$$
\Delta P_{r}=\left[\frac{\gamma\left(B-\sqrt{B^{2}-4 A C}\right)}{(\gamma+1) A}-\frac{\gamma-1}{\gamma+1}\right]\left(\Delta P_{f}+P_{0}\right)-P_{0}
$$


The reflection overpressure is a function of incident overpressure and incident angle.

$$
\Delta P_{r}=f\left(\Delta P_{f}, \omega\right)
$$

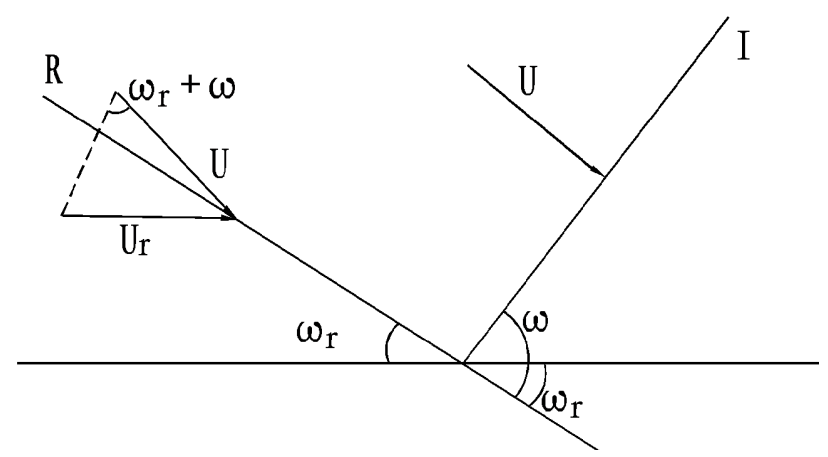

Figure 1: Incident shock wave and reflection wave on the ground.

The sonic angle of regular reflection is defined as the incident angle where the perturbation after the reflected airblast wave catches up with the intersecting point of the incident wave and reflected wave. The sonic angle $\omega$ can be calculated with the following formulae.

$$
\begin{gathered}
U+C=\frac{U}{\sin \omega} \\
\sigma^{\prime 6}-\left[3+\frac{2 \gamma\left(\sigma^{2}-1\right)^{2} \cos ^{2} \omega}{(\gamma+1) \sigma^{2}}\right] \sigma^{\prime 4} \\
+\left[3+\frac{(\gamma-1)\left(\sigma^{2}-1\right)^{2} \cos ^{2} \omega}{(\gamma+1) \sigma^{2}}\right] \sigma^{\prime 2}-1=0
\end{gathered}
$$

\subsection{The horizontal dynamic pressure of regular reflection}

The dimensional formula for calculating the horizontal dynamic pressure of shock waves from regular reflection is given below.

$$
q_{r}=\frac{1}{2} \rho_{r} U_{r}^{2}
$$

The projection of the incident and reflected shock wave particle velocity on the reflected shock satisfies the equality conditions. Then the dynamic pressure can be obtained.

$$
U_{r} \cos \omega_{r}=U \sin \left(\omega+\omega_{r}\right)
$$




$$
q_{r}=\frac{1}{2} \rho_{0} \eta_{r} \cdot \eta \cdot C^{2}(\tau-\sigma)^{2} \frac{\sin ^{2}\left(\omega+\omega_{r}\right)}{\cos ^{2} \omega_{r}}
$$

Utilizing Newton's binary iteration, the sonic angle or the relation between the reflection overpressure and the incident overpressure can be found out.

$$
\begin{gathered}
A_{0} \sigma_{r 1}^{2}-\left(2 A_{0}-B_{0} \cos ^{2} \omega++C_{0} \cot ^{2} \omega\right) \sigma_{r 1} \\
+\left(A_{0}-D_{0} \cos ^{2} \omega+E_{0} \cot ^{2} \omega\right)=0 \\
\sigma_{r 1}^{3}-\left(3+A_{s} \cos ^{2} \omega\right) \sigma_{r 1}^{2}+\left(3+B_{s} \cos ^{2} \omega\right) \sigma_{r 1}-1=0 \\
\sigma_{r s}^{n+1}=\sigma_{r s}{ }^{n}+\left|\begin{array}{cc}
F_{1}^{n} & \left(\frac{\partial F_{1}}{\partial \omega}\right)^{n} \\
F_{2}^{n} & \left(\frac{\partial F_{2}}{\partial \omega}\right)^{n}
\end{array}\right| /\left|\begin{array}{ll}
\left(\frac{\partial F_{1}}{\partial \sigma_{r 1}}\right)^{n} & \left(\frac{\partial F_{1}}{\partial \omega}\right)^{n} \\
\left(\frac{\partial F_{2}}{\partial \sigma_{r 1}}\right)^{n} & \left(\frac{\partial F_{2}}{\partial \omega}\right)^{n}
\end{array}\right| \\
\omega_{s}^{n+1}=\omega_{s}{ }^{n}+\left|\begin{array}{ll}
\left(\frac{\partial F_{1}}{\partial \sigma_{r 1}}\right)^{n} & F_{1}^{n} \\
\left(\frac{\partial F_{2}}{\partial \sigma_{r 1}}\right)^{n} & F_{2}^{n}
\end{array}\right| /\left|\begin{array}{ll}
\left(\frac{\partial F_{1}}{\partial \sigma_{r 1}}\right)^{n} & \left(\frac{\partial F_{1}}{\partial \omega}\right)^{n} \\
\left(\frac{\partial F_{2}}{\partial \sigma_{r 1}}\right)^{n} & \left(\frac{\partial F_{2}}{\partial \omega}\right)^{n}
\end{array}\right|
\end{gathered}
$$

Hence the maximum sonic angle can be found out to be $46^{\circ}$.

$$
\begin{gathered}
\omega_{s}=f(\Delta P) \\
\Delta P_{r s}=f(\Delta P)
\end{gathered}
$$

Table 2: Regular reflection overpressure coefficient.

\begin{tabular}{|c|c|c|c|c|c|c|c|c|}
\hline $\begin{array}{c}\Delta \mathrm{P}_{\mathrm{f}} \\
(\mathrm{MPa})\end{array}$ & $\gamma$ & $\omega=0^{\circ}$ & $\omega=10^{\circ}$ & $\omega=20^{\circ}$ & $\omega=30^{\circ}$ & $\omega=40^{\circ}$ & $\omega=\omega_{\text {ex }}$ & $\left.\omega_{\text {ex }}{ }^{\circ}\right]$ \\
\hline 0.5 & 1.400 & $4.48^{*}$ & $4.41^{*}$ & $4.24^{*}$ & $4.03^{*}$ & & $4.54^{*}$ & $39.25^{*}$ \\
\hline 1 & 1.400 & $5.51^{*}$ & $5.41^{*}$ & $5.16^{*}$ & $4.82^{*}$ & & $5.22^{*}$ & $39.31^{*}$ \\
\hline 2 & 1.390 & $6.50^{*}$ & $6.38^{*}$ & $6.04^{*}$ & $5.58^{*}$ & & $5.89^{*}$ & $39.72^{*}$ \\
\hline 5 & 1.350 & $7.80^{*}$ & $7.52^{*}$ & $7.08^{*}$ & $6.45^{*}$ & $6.10^{*}$ & $5.53^{*}$ & $40.91^{*}$ \\
\hline 10 & 1.300 & $9.04^{*}$ & $8.84^{*}$ & $8.28^{*}$ & $7.46^{*}$ & $6.70^{*}$ & $7.13^{*}$ & $42.43^{*}$ \\
\hline 20 & 1.260 & $10.28^{*}$ & $10.04^{*}$ & $9.36^{*}$ & $8.36^{*}$ & $7.30^{*}$ & $7.60^{*}$ & $43.85^{*}$ \\
\hline 50 & 1.220 & $11.86^{*}$ & $11.58^{*}$ & $10.75^{*}$ & $9.52^{*}$ & $8.13^{*}$ & $8.08^{*}$ & $45.47^{*}$ \\
\hline 70 & 1.210 & $12.35^{*}$ & $12.04^{*}$ & $11.18^{*}$ & $9.88^{*}$ & $8.40^{*}$ & $8.24^{*}$ & $45.92^{*}$ \\
\hline 100 & 1.200 & $12.86^{*}$ & $12.55^{*}$ & $11.63^{*}$ & $10.27 \mathrm{v}$ & 8.68 & $8.43^{*}$ & $46.40^{*}$ \\
\hline
\end{tabular}


Table 3: $\quad$ Reflection overpressure.

\begin{tabular}{|c|c|c|c|c|c|c|}
\hline$\Delta \mathrm{P}_{\mathrm{f}}(\mathrm{MPa})$ & $\omega=40^{\circ}$ & $\omega=50^{\circ}$ & $\omega=60^{\circ}$ & $\omega=70^{\circ}$ & $\omega=80^{\circ}$ & $\omega=90^{\circ}$ \\
\hline 0.5 & $4.48^{*}$ & $3.72^{*}$ & $3.10^{*}$ & $2.58^{*}$ & $2.15^{*}$ & $1.79^{*}$ \\
\hline 1 & $5.14^{*}$ & $4.19^{*}$ & $3.41^{*}$ & $2.78^{*}$ & $2.26^{*}$ & $1.84^{*}$ \\
\hline 2 & $5.85^{*}$ & $4.67^{*}$ & $3.72^{*}$ & $2.96^{*}$ & $2.36^{*}$ & $1.88^{*}$ \\
\hline 5 & & $5.24^{*}$ & $4.08^{*}$ & $3.17^{*}$ & $2.47^{*}$ & $1.92^{*}$ \\
\hline 10 & & $5.79^{*}$ & $4.41^{*}$ & $3.35^{*}$ & $2.55^{*}$ & $1.94^{*}$ \\
\hline 20 & & $6.34^{*}$ & $4.73^{*}$ & $3.52^{*}$ & $2.62^{*}$ & $1.96^{*}$ \\
\hline 50 & & $7.01^{*}$ & $5.10^{*}$ & $3.71^{*}$ & $2.70^{*}$ & $1.97^{*}$ \\
\hline 100 & & $7.55^{*}$ & $5.56^{*}$ & $4.09^{*}$ & $3.01^{*}$ & $2.22^{*}$ \\
\hline
\end{tabular}

\section{Non-regular reflection for bursts in air}

\subsection{The starting angle of mach reflection and transition reflection}

The Mach reflection starting angle meets the requirements of low-stem Mach reflection - the reflection wave catches up with the intersection point of the incident wave and reflection wave, and then the combined low-stem Mach shock wave perpendicular to the ground is formed (see Fig.2).

$$
\sin ^{2} \omega_{M}=\frac{2 \gamma P_{0}+(\gamma+1) \Delta P_{f}}{2 \gamma P_{0}+(\gamma+1) \Delta P_{r M}}
$$

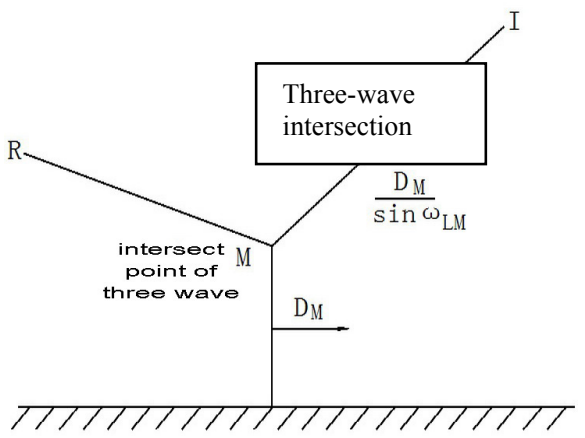

Figure 2: $\quad$ The starting angle of Mach reflection and transition reflection. 
The sonic angle and the transition overpressure can be obtained by using the regular reflection equation, the low-stem Mach reflection angle equation, and the sonic angle equation.

$$
\begin{gathered}
A_{0} \sigma_{r}^{4}-\left(2 A_{0}+B_{0} \cos ^{2} \omega+C_{0} \cot ^{2} \omega\right) \sigma_{r}^{2} \\
+\left(A_{0}-D_{0} \cos ^{2} \omega\right)+E_{0} \cot ^{2} \omega=0 \\
\sigma_{r}^{6}-\left(3+A_{S} \cos ^{2} \omega\right) \sigma_{r}^{4}+\left(3+B_{S} \cos ^{2} \omega\right) \sigma_{r}^{2}-1=0 \\
2 r(r+1)\left(\sigma_{r}^{2}-\sigma^{2}\right) \sin ^{2} \omega-2 r\left[2 r \sigma^{2}-(r-1)\right] \cos ^{2} \omega \\
-2 r(r+1)\left(1-\sigma^{2}\right)=0 \\
\omega^{n+1}=\omega^{n}+\left|\begin{array}{lll}
\frac{\partial F_{1}}{\partial \sigma_{r}} & \frac{\partial F_{1}}{\partial \sigma} & F_{1} \\
\frac{\partial F_{2}}{\partial \sigma_{r}} & \frac{\partial F_{2}}{\partial \sigma} & F_{2} \\
\frac{\partial F_{3}}{\partial \sigma_{r}} & \frac{\partial F_{3}}{\partial \sigma} & F_{3}
\end{array}\right| / /
\end{gathered}
$$

And the maximum incident overpressure $\Delta \mathrm{P}_{\max }$ is:

$$
\Delta P_{\text {max }}=0.1688 M P a
$$

\subsection{The mach reflection area}

The unified calculation of the overpressure of the Mach reflection area is based on Russian scholar Korotkov's trinomial theory, and the boundaries are controlled by the sonic angle, the low-stem Mach reflection starting angle conditions and the semi-spherical reflection conditions respectively.

$$
\Delta P_{r}=\Delta P\left(a_{p}+b_{p} \alpha+c_{p} \alpha^{3}\right)
$$

When $\Delta P \geq \Delta P_{\max }$,

$$
C_{p}=\left(\frac{\Delta P_{r s}}{\Delta P_{f}}-a_{p}-b_{p} \cdot \alpha_{s}\right) \frac{1}{\alpha_{s}^{3}} \text { (Sonic angle condition) }
$$

So the overpressure of the Mach reflection area is determined by the sonic angle conditions. When $\Delta P<\Delta P_{\max }$,

$$
C_{p}=\left(\frac{\Delta P_{r L}}{\Delta P_{f}}-a_{p}-b_{p} \cdot \alpha_{L M}\right) \frac{1}{\alpha_{L M}^{3}} \text { (Low stem Mach condition) }
$$


which means the overpressure of the Mach reflection area is determined by the sonic angle condition and low-stem Mach reflection conditions. $a_{\mathrm{p}}$ is calculated under semi-spherical conditions, and $b_{\mathrm{p}}$ is calculated based on Lighthill's smallangle shock reflection linearization theory's analytic solution.

Let $M_{f 0}$ be the shock wave Mach number when the air particle flow $\mathrm{M}_{\mathrm{u}}=1$.

When $M<M_{f 0}$, for subsonic flow,

$$
\begin{aligned}
& b_{p}=\frac{2 D_{1} C_{1}}{K(\alpha-\beta)}\left[\frac{\pi}{2}\left(\frac{1}{T}-\frac{1}{S}\right)-\left(\frac{\operatorname{arctg} \frac{\sqrt{2}}{T}}{T}-\frac{\operatorname{arctg} \frac{\sqrt{2}}{S}}{S}\right)\right]+ \\
& \frac{C_{1}\left(\pi-2 \operatorname{arctg} \frac{\sqrt{2}}{T}\right)}{K(\alpha-\beta)\left(\beta^{2}-\lambda^{2}\right) T}-\frac{C_{1}\left(\pi-2 \operatorname{arctg} \frac{\sqrt{2}}{S}\right)}{K(\alpha-\beta)\left(\alpha^{2}-\lambda^{2}\right) S} \\
& -\frac{C_{1}(\alpha+\beta) L_{n}\left[\left(1+\sqrt{\frac{2-\lambda^{2}}{2}}\right) /\left(1-\sqrt{\frac{2-\lambda^{2}}{2}}\right)\right]}{K\left(\alpha^{2}-\lambda^{2}\right)\left(\beta^{2}-\lambda^{2}\right) \sqrt{2-\lambda^{2}}}
\end{aligned}
$$

When $M=M_{f 0}$, for equal sonic flow,

$$
\begin{aligned}
& b_{p}=\frac{2 D_{1} C_{1}}{K(\alpha-\beta)}\left[\frac{\pi}{2}\left(\frac{1}{T}-\frac{1}{S}\right)-\left(\frac{\operatorname{arctg} \frac{\sqrt{2}}{T}}{T}-\frac{\operatorname{arctg} \frac{\sqrt{2}}{S}}{S}\right)\right] \\
& +\frac{\pi C_{1}}{K(\alpha-\beta)}\left(\frac{1}{T^{3}}-\frac{1}{S^{3}}\right)-\frac{\sqrt{2} C_{1}(\alpha+\beta)}{K S^{2} T^{2}}-\frac{2 C_{1}}{K(\alpha-\beta)}\left(\frac{\operatorname{arctg} \frac{\sqrt{2}}{T}}{T^{3}}-\frac{\operatorname{arctg} \frac{\sqrt{2}}{S}}{S^{3}}\right)
\end{aligned}
$$

When $M>M_{f 0}$, for supersonic flow,

$$
\begin{aligned}
& b_{p}=\frac{2 D_{1} C_{1}}{K(\alpha-\beta)}\left[\frac{\pi}{2}\left(\frac{1}{T}-\frac{1}{S}\right)-\left(\frac{\operatorname{arctg} \frac{\sqrt{2}}{T}}{T}-\frac{\operatorname{arctg} \frac{\sqrt{2}}{S}}{S}\right)\right] \\
& +\frac{C_{1}\left(\pi-2 \operatorname{arctg} \frac{\sqrt{2}}{T}\right)}{K(\alpha-\beta)\left(\beta^{2}-\lambda^{2}\right) T}-\frac{C_{1}\left(\pi-2 \operatorname{arctg} \frac{\sqrt{2}}{T}\right)}{K(\alpha-\beta)\left(\beta^{2}-\lambda^{2}\right) S}-\frac{C_{1}(\alpha+\beta)\left(\pi-2 \operatorname{arctg} \sqrt{\frac{2}{\lambda^{2}-2}}\right)}{K\left(\alpha^{2}-\lambda^{2}\right)\left(\beta^{2}-\lambda^{2}\right) \sqrt{\lambda^{2}-2}}
\end{aligned}
$$




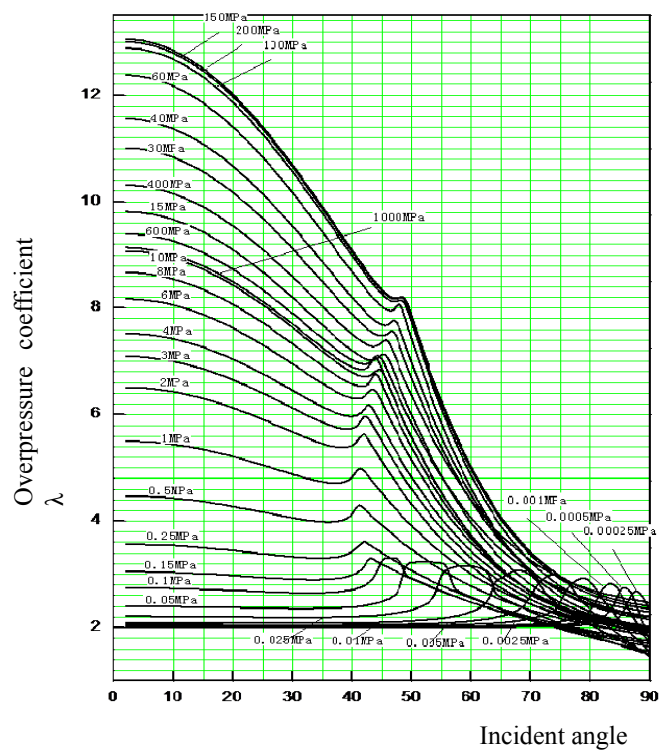

Figure 3: Reflection overpressure coefficient versus incident overpressure and incident angle.

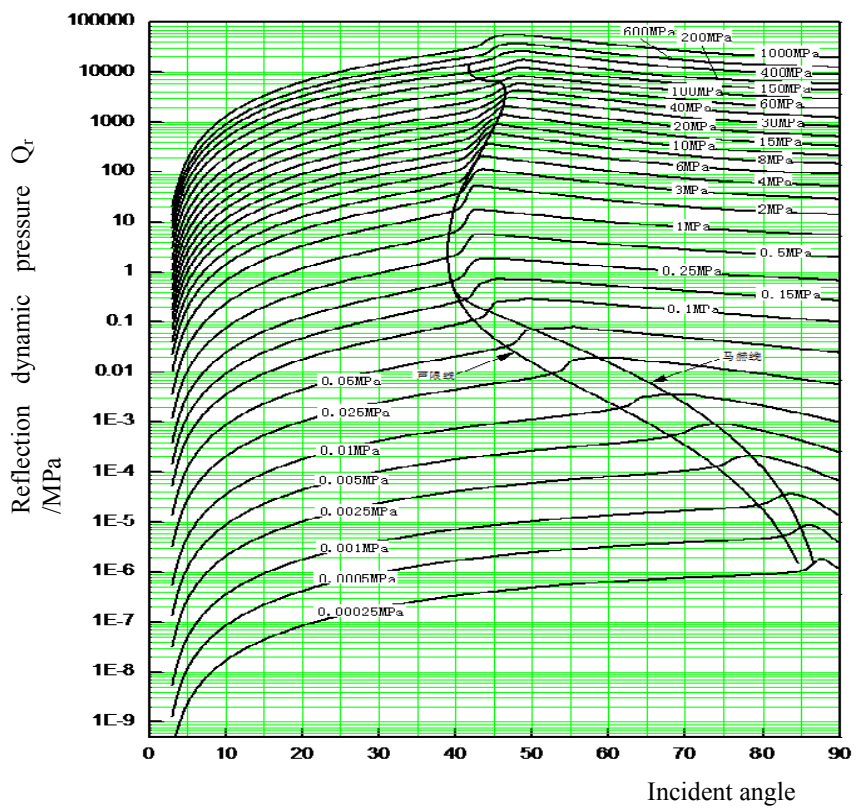

Figure 4: Reflection dynamic pressure versus incident angle and incident overpressure. 


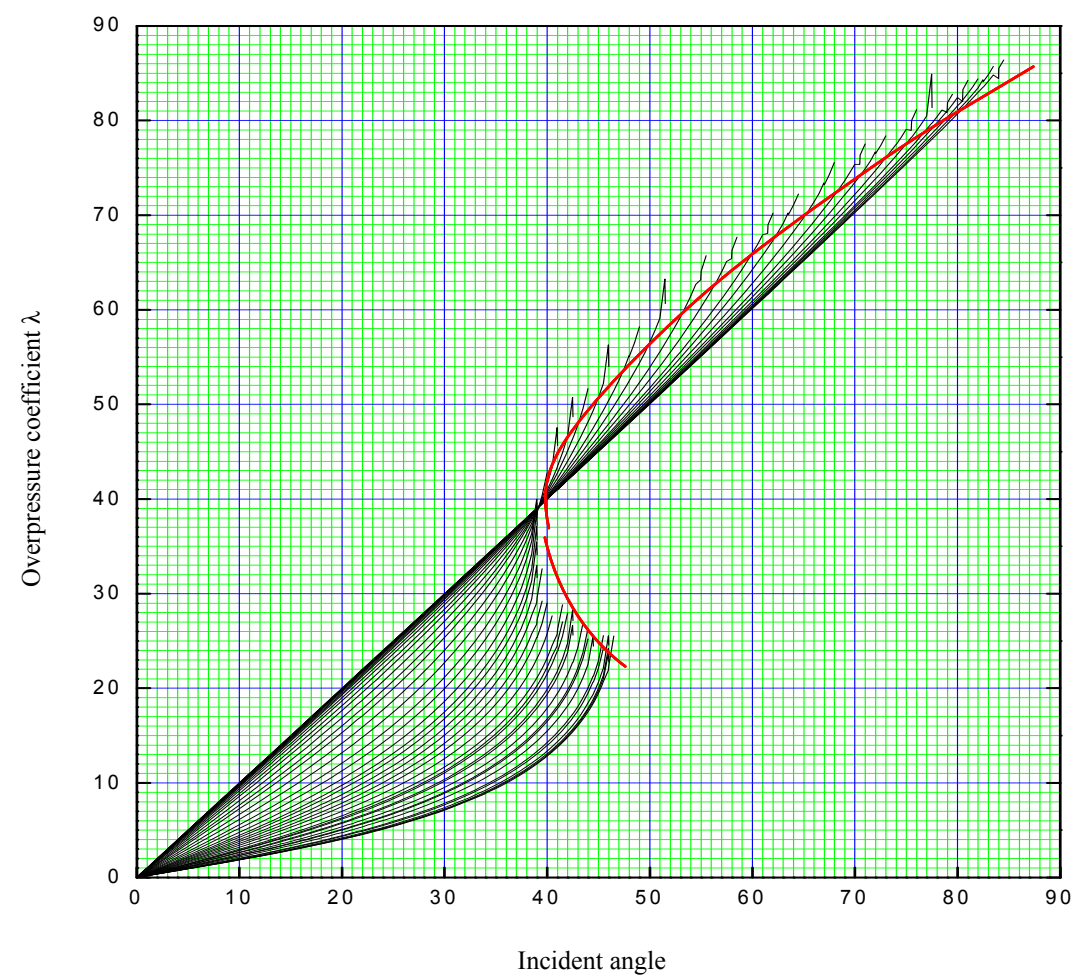

Figure 5: Relation between incident angle and reflection angle.

\section{Conclusion}

With real-air state equations, the calculation of regular reflection and Mach reflection of shock waves are studied in depth. For regular reflection, based on the variable- $\gamma$ reflection theory, the reflection termination is under the control of the sonic angle. For Mach reflection, Russian scholar Korotkov's method is adopted to apply the theoretical linear solution with small slope angles, and to find out the conditions for Mach reflection starting and for hemispherical reflection termination. Our study leads to a curve diagram showing the relation of ground shock wave's reflection overpressure and dynamic pressure versus incident overpressure and incident angle. Our results are available to research on the reflection of shock waves from different types of bursts in real air.

\section{References}

[1] Brode, H.L., Point source explosion in air. The RAND Corporation, Research Memorandum RM-1824-AEC, December 3, 1956. 
[2] Brode, H. L., A calculation of the blast wave from a spherical charge of TNT, The RAND Corporation, Research Memorandum RM-1965, October 21, 1957. Phys. Fluids, 2, pp. 217, 1959.

[3] Taylor, G. I., Proc. Roy. Soc., 201A, pp. 159-186, 1950.

[4] Shock Hydrodynamics and blast wave. Los Alamos Scientific Laboratory, Report AECD-2860, 1944.

[5] Glasstone, Samuel (ed.), The effects of nuclear weapons. U.S. Department of Defense, U.S. Atomic Energy Commission, June 1957.

[6] William E. Baker., Explosions in air. University of Texas Press, Austin, 1973.

[7] Jon A.B. and Scot Tumlin., Extremely explosive: Explosion effects. 3D design, 4(8): 74-80, August 1998.

[8] Hornung H.G. and Robinson M. L., Transition from regular to Mach reflection of shock-wave. Part 2. The steady-flow criterion. Journal of Fluid Mechanics, 123, pp. 155-164, 1982. 